\title{
Measuring the Weak Mixing Angle in the DUNE Near-Detector Complex
}

\author{
André de Gouvêa $\odot,{ }^{1}$ Pedro A. N. Machado, ${ }^{2}$ Yuber F. Perez-Gonzalez $\odot,{ }^{1,2,3}$ and Zahra Tabrizi ${ }^{4}$ \\ ${ }^{1}$ Department of Physics \& Astronomy, Northwestern University, Evanston, Illinois 60208, USA \\ ${ }^{2}$ Theoretical Physics Department, Fermilab, P.O. Box 500, Batavia, Illinois 60510, USA \\ ${ }^{3}$ Colegio de Física Fundamental e Interdisciplinaria de las Américas (COFI), 254 Norzagaray street, San Juan 00901, Puerto Rico \\ ${ }^{4}$ Instituto de Física Gleb Wataghin, Universidade Estadual de Campinas (UNICAMP), \\ Rua Sérgio Buarque de Holanda, 777, Campinas, SP 13083-859, Brazil
}

(Received 14 February 2020; revised 3 June 2020; accepted 7 July 2020; published 29 July 2020)

\begin{abstract}
The planned DUNE experiment will have excellent sensitivity to the vector and axial couplings of the electron to the $Z$ boson via precision measurements of neutrino-electron scattering. We investigate the sensitivity of DUNE-PRISM, a movable near detector in the direction perpendicular to the beam line, and find that it will qualitatively impact our ability to constrain the weak couplings of the electron. We translate these neutrino-electron scattering measurements into a determination of the weak mixing angle at low scales and estimate that, with seven years of data taking, the DUNE near detector can be used to measure $\sin ^{2} \theta_{W}$ with about $2 \%$ precision. We also discuss the impact of combining neutrino-electron scattering data with neutrino trident production at DUNE-PRISM.
\end{abstract}

DOI: 10.1103/PhysRevLett.125.051803

The standard model of particle physics (SM) is a quantum field theory with a $\mathrm{SU}(3)_{c} \times \mathrm{SU}(2)_{L} \times \mathrm{U}(1)_{Y}$ gauge symmetry, corresponding to the color, weak-isospin, and hypercharge interactions, respectively, along with a set of fermion and boson fields describing the particles observed in nature. Free SM parameters - the gauge and Yukawa couplings, together with the scalar potential parameters - need to be determined by comparing the results of theoretical computations to a finite set of the experimental measurements.

The weak mixing angle $\theta_{W}$ (or, more precisely, its sine-squared, $\sin ^{2} \theta_{W}$ ) parametrizes several measurable quantities: the mass ratio of the weak gauge bosons, some weak-interaction cross sections, and parity-violating observables. It is a crucial ingredient of the electroweak precision observables, a set of experimental observables designed to test the SM internal consistency.

The exact definition of the weak mixing angle depends on the renormalization scheme, that is, the convention of which quantities are taken as input and which are derived from these inputs, along with the recipe for handling quantum corrections. As quantum corrections are relevant, $\sin ^{2} \theta_{W}$ depends on the scale at which it is being measured. For example, in the modified minimal subtraction scheme $[1,2], \overline{\mathrm{MS}}$, the weak mixing angle is

Published by the American Physical Society under the terms of the Creative Commons Attribution 4.0 International license. Further distribution of this work must maintain attribution to the author(s) and the published article's title, journal citation, and DOI. Funded by SCOAP .

$$
\sin ^{2} \theta_{W}(\mu) \equiv \frac{g^{\prime 2}(\mu)}{g^{2}(\mu)+g^{\prime 2}(\mu)},
$$

where $g$ and $g^{\prime}$ are the $\mathrm{SU}(2)_{L}$ and $\mathrm{U}(1)_{Y}$ gauge coupling constants, respectively, and $\mu$ is the scale of the physical process under consideration. The SM predicts, under a specific renormalization scheme, a unique scale dependence for $\sin ^{2} \theta_{W}$. This dependence has been confirmed by precise measurements at very different energy scales, including atomic parity violation, electron-proton scattering, Möller scattering, neutrino-nucleus and neutrino-electron scattering, electron deep-inelastic scattering, and the $Z$ - and $W$-boson masses (see Ref. [3] for a comprehensive review).

The $\mathrm{NuTeV}$ result [4], the most precise measurement of $\sin ^{2} \theta_{W}$ using neutrino scattering, stands out from the other measurements. Considering the ratios of neutral-current to charged-current and neutrino-nucleus to antineutrinonucleus cross sections, they find $\sin ^{2} \theta_{W}=0.2407 \pm$ 0.0016 (in the $\overline{\mathrm{MS}}$ scheme) at an average energy scale $\langle\mu\rangle \simeq 4.5 \mathrm{GeV}$. This measurement deviates from the SM expectation anchored by the more precise measurements at LEP [5] at the $3 \sigma$ level. Effects inherent to the intricacies of neutrino-nucleus scattering, potentially unaccounted for or only partially accounted for by the collaboration, have been identified as candidate sources for the discrepancy [6-24]. A definitive answer remains elusive. Regardless, it stands to reason that other precise measurements of $\sin ^{2} \theta_{W}$ using neutrino scattering will help shed light on the situation.

Next-generation neutrino experiments like LBNFDUNE [25] and T2HK [26] will include very intense neutrino beams with energies that range from several 
hundred $\mathrm{MeV}$ to several $\mathrm{GeV}$. The neutrino-nucleus scattering cross sections, at these energies, have large uncertainties due to nuclear and nonperturbative effects [27], making it very challenging to use them to infer $\sin ^{2} \theta_{W}$. Neutrino-electron scattering, on the other hand, provides a more promising environment [28-32]. Even in this case, however, one still needs to address significant challenges. First, the cross section for neutrino-electron scattering is 3 orders of magnitude smaller than that for neutrino-nucleus scattering, translating into poor statistics in most neutrino experiments. Second, while the neutrino-electron cross section depends mostly on $\sin ^{2} \theta_{W}$, the neutrino beam originates from the in-flight decay of charged mesons produced by high-energy protons hitting a fixed target. First-principles computations of the meson production rate and kinematics are not possible and one must rely on phenomenological models and experimental data; uncertainties on the overall neutrino flux and energy distribution are at the $5 \%$ to $15 \%$ level [33-35].

Near-detector complexes are designed to circumvent some of the large uncertainties in the flux and cross sections and allow precision measurements of neutrino oscillations [35]. DUNE-PRISM [36], currently part of the LBNF-DUNE proposal, is a near detector that is capable of moving in the direction perpendicular to the neutrino-beam axis. Although the neutrino flux has prohibitively large uncertainties, the ratios of on-axis to off-axis fluxes are dictated only by meson-decay kinematics and thus are much better understood. Therefore, measurements of the neutrinoelectron-scattering spectrum at different off-axis positions should allow an unprecedented measurement of the weak mixing angle with neutrinos.

In general terms, the neutrino-electron scattering cross section depends on the vector and axial couplings, $g_{V}$ and $g_{A}$, between the $Z$ boson and the electron (see CHARM-II [37], LSND [38], and TEXONO [39]). We will estimate the DUNE-PRISM sensitivity to such parameters via neutrinoelectron scattering data. A hidden but very safe assumption is that the cross section depends only on the neutrino lefthanded coupling to the $Z$ boson. The reason for this is that all neutrinos and antineutrinos used in neutrino scattering are produced in charged-current processes $\left(\pi^{+} \rightarrow \mu^{+} \nu_{\mu}\right.$, $n \rightarrow p e \bar{\nu}_{e}, D_{s} \rightarrow \tau^{+} \nu_{\tau}$, etc.) and are, to a very good precision, $100 \%$ polarized. Lepton-collider data, combined with those from neutrino-electron scattering, for example, can be used to determine the right-handed coupling of neutrinos to the $Z$ boson [40].

The differential cross section for a neutrino with flavor $\alpha=e, \mu, \tau$ to scatter off an electron at rest is

$$
\begin{aligned}
\frac{d \sigma}{d E_{R}} & =\frac{2 G_{F}^{2} m_{e}}{\pi}\left\{g_{1}^{2}+g_{2}^{2}\left(1-\frac{E_{R}}{E_{\nu}}\right)^{2}-g_{1} g_{2} \frac{m_{e} E_{R}}{E_{\nu}^{2}}\right\} \\
& \simeq 1.72 \times 10^{-41}\left\{g_{1}^{2}+g_{2}^{2}\left(1-\frac{E_{R}}{E_{\nu}}\right)^{2}\right\} \frac{\mathrm{cm}^{2}}{\mathrm{GeV}},
\end{aligned}
$$

TABLE I. Couplings $g_{1}$ and $g_{2}$ [see Eq. (2)] as a function of the electron-Z-boson couplings $g_{V}$ and $g_{A}$, for each neutrino flavor, along with the corresponding SM value. $s_{W}^{2} \equiv \sin ^{2} \theta_{W}$.

\begin{tabular}{lcccc}
\hline \hline$\nu_{\alpha}$ & $g_{1}$ & $g_{1}(\mathrm{SM})$ & $g_{2}$ & $g_{2}(\mathrm{SM})$ \\
\hline$\nu_{e}$ & $1+\left(g_{V}+g_{A}\right) / 2$ & $1 / 2+s_{W}^{2}$ & $\left(g_{V}-g_{A}\right) / 2$ & $s_{W}^{2}$ \\
$\nu_{\mu, \tau}$ & $\left(g_{V}+g_{A}\right) / 2$ & $-1 / 2+s_{W}^{2}$ & $\left(g_{V}-g_{A}\right) / 2$ & $s_{W}^{2}$ \\
$\bar{\nu}_{e}$ & $\left(g_{V}-g_{A}\right) / 2$ & $s_{W}^{2}$ & $1+\left(g_{V}+g_{A}\right) / 2$ & $1 / 2+s_{W}^{2}$ \\
$\bar{\nu}_{\mu, \tau}$ & $\left(g_{V}-g_{A}\right) / 2$ & $s_{W}^{2}$ & $\left(g_{V}+g_{A}\right) / 2$ & $-1 / 2+s_{W}^{2}$ \\
\hline \hline
\end{tabular}

where $G_{F}$ is the Fermi constant, $E_{\nu}$ is the incoming neutrino energy, and $m_{e}$ and $E_{R}$ are the electron mass and recoil kinetic energy, respectively.

The couplings $g_{1}$ and $g_{2}$ depend on the neutrino flavor and can be written in terms of $g_{V}$ and $g_{A}$; thus, they can be expressed in terms of $\sin ^{2} \theta_{W}$, see Table I. More generally, if $g_{V}$ and $g_{A}$ are considered to be free parameters, they can be independently extracted from the recoil-electron energy spectrum so data from DUNE-PRISM are expected to constrain nontrivial regions in the $g_{V} \times g_{A}$ plane.

Strictly speaking, the neutrino-electron cross section is also subject to quantum corrections that will introduce additional dependence on $Q^{2} \equiv 2 E_{R} m_{e} \quad[41,42]$. Kinematics dictates that the maximum recoil energy is approximately $E_{R}^{\max } \simeq E_{\nu}-m_{e} / 2$. Because of kinematics and the energy profile of DUNE's neutrino flux, most electron recoil events will lie within $0.2 \lesssim E_{R} \lesssim 10 \mathrm{GeV}$. Therefore, the $Q^{2}$ values accessible to DUNE are, roughly, in the range $(10-100 \mathrm{MeV})^{2}$, where loop corrections to $\sin ^{2} \theta_{W}$ have little scale dependence [3,43]. Thus, by analyzing the $Q^{2}$ distribution in detail, the couplings in Eq. (2) can be interpreted as the renormalized couplings in the $\overline{\mathrm{MS}}$ scheme at an average scale $\left\langle Q^{2}\right\rangle=(55 \mathrm{MeV})^{2}$.

Assuming the $\mathrm{SM}$, the cross section for $\nu_{\mu}-e$ scattering is

$$
\frac{d \sigma}{d E_{R}} \propto\left(\frac{1}{4}-\sin ^{2} \theta_{W}\right)+\sin ^{4} \theta_{W}\left(2-\frac{2 E_{R}}{E_{\nu}}+\frac{E_{R}^{2}}{E_{\nu}^{2}}\right) .
$$

Since $\sin ^{2} \theta_{W}$ is close to $1 / 4$, the first term is suppressed relative to the second one. This implies that the value of $\sin ^{2} \theta_{W}$, to leading order, modifies the overall normalization of the $\nu_{\mu}-e$-scattering cross section and the effect of changing $\sin ^{2} \theta_{W}$ is nearly degenerate with that of changing the overall normalization of the $\nu_{\mu}$ flux. The situation is different for $\nu_{e}-e$ and $\bar{\nu}_{e}-e$ scattering; $\sin ^{2} \theta_{W}$ has a significant impact on the shape of the recoil-electron energy distributions. It turns out, unfortunately, that, at DUNE, the neutrino flux is dominated by $\nu_{\mu}$ and the $\nu_{e}$ contribution is relatively small, around a few percent.

In this context, DUNE-PRISM is expected to provide nontrivial information. In accelerator neutrino experiments, the $\nu_{\mu}$ comes predominantly from the two-body decay $\pi^{+} \rightarrow \mu^{+} \nu_{\mu}$ (and $K^{+} \rightarrow \mu^{+} \nu_{\mu}$, to a lesser extent) while the 


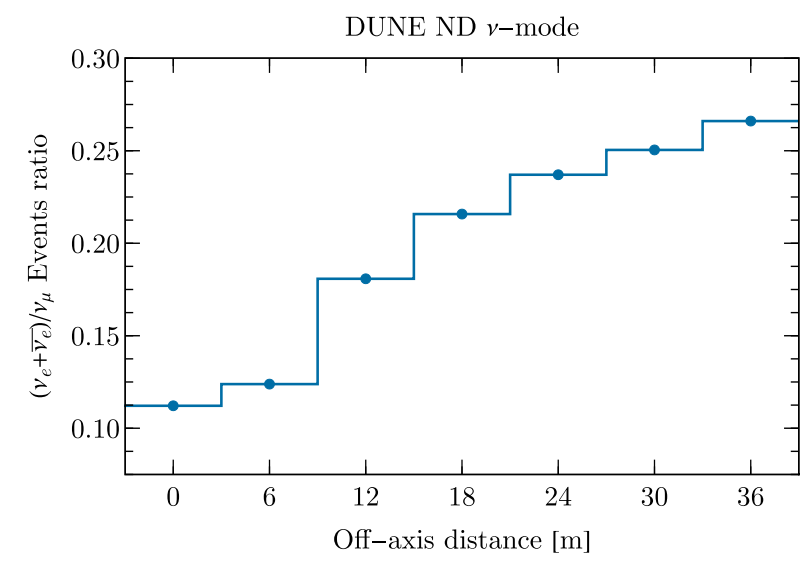

FIG. 1. Ratio of the number of events expected from $\nu_{e}-e+$ $\bar{\nu}_{e}-e$ scattering to that of $\nu_{\mu}-e$ scattering, in neutrino-mode running, as a function of the off-axis distance.

$\nu_{e}$ comes from the three-body decays of kaons and muons. For the same parent energy, the flux of $\nu_{e}$ has a larger angular spread than that of $\nu_{\mu}$ so the off-axis $\nu_{e}$ to $\nu_{\mu}$ flux ratio is larger than the on-axis one.

To estimate how well DUNE-PRISM can contribute to the precision electroweak physics program, we compute the sensitivity to $\sin ^{2} \theta_{W}$ for both on-axis and off-axis runnings. For concreteness, we assume seven years of data taking equally divided between the neutrino and antineutrino modes. We assume a 75 ton fiducial mass liquid argon time projection chamber (LArTPC) and a 1.2 MW proton beam, as described in the DUNE Conceptual Design Report [25]. For the off-axis configuration, we assume the near detector will take data at seven different positions, half of the time on axis and half of the time equally divided in the off-axis positions. The detector is assumed to be $574 \mathrm{~m}$ away from the source in the beam axis direction while its transverse distances to the beam axis are $6 \mathrm{~N}$ meters, $N=0, \ldots 6$. The detector experiences at each position a flux that is approximately $10 \mathrm{~N}$ mrad off axis, respectively. Figure 1 depicts the ratio of the number of events expected from $\nu_{e}-e$ and $\bar{\nu}_{e}-e$ scattering to that of $\nu_{\mu}-e$ scattering, in neutrino-mode running, as a function of the off-axis distance. As expected, the relevance of the $\nu_{e}$ and $\bar{\nu}_{e}$-initiated events grows significantly with the off-axis angle. Note that, while the flux ratio is of order a few percent, the $\nu_{e}-e$-scattering cross section is larger than the $\nu_{\mu}-e$ so, even on axis, the $\nu_{e}$ contribution is of order $10 \%$.

To account for the energy-dependent neutrino-flux uncertainties and the correlations between the fluxes at different off-axis angles, we make use of a covariance matrix spanning all DUNE-PRISM positions and neutrino flavors, derived from detailed simulations of hadron production in the beam target followed by magnetic-horn focusing of charged particles [44]. The binning is performed in $E_{e} \theta_{e}^{2}$, where $E_{e}=E_{R}+m_{e}$ is the total electron energy and $\theta_{e}$ is the electron scattering angle relative to the beam direction (see Supplemental Material [45] for details.). We consider a threshold kinetic energy $E_{R}>50 \mathrm{MeV}$ and perform the analysis in the range $\left(0.05<E_{R}<20\right) \mathrm{GeV}$.

The main backgrounds for neutrino-electron scattering are charged-current quasielastic (CCQE) $\nu_{e}$-scattering events, $\nu_{e} A \rightarrow e^{-} A^{\prime}$ and misidentified $\pi^{0}$ events with no detectable hadronic activity, $\nu A \rightarrow \nu \pi^{0} A$. Although the $\nu_{e}$ flux is only a few percent of the total neutrino flux, the CCQE cross section is over 1000 times larger than that for neutrino-electron scattering. We simulate these backgrounds using the NuWro event generator [46], and allow a $10 \%$ normalization uncertainty of both of them. We cut any event which has at least one proton with kinetic energy above $50 \mathrm{MeV}$. For the $\pi^{0}$ background, we also require one photon to be soft, below $30 \mathrm{MeV}$, to be accepted. In principle, if the photons are sufficiently collinear, the two showers could be misidentified as an electron event. As the minimum photonphoton opening angle is $\theta>15.5^{\circ}\left(\mathrm{GeV} / E_{\pi^{0}}\right)$, it is unlikely that this poses a background and therefore we have neglected it.

Kinematics limit $E_{e} \theta_{e}^{2}<2 m_{e}$ for neutrino-electron scattering and thus we bin on $E_{e} \theta_{e}^{2}$ to improve background rejection. LArTPCs have an exquisite angular resolution, of order $1^{\circ}$, for electromagnetic showers [25]. In the Supplemental Material [45], we show how angular resolution affects the $E_{e} \theta_{e}^{2}$ spectrum and the sensitivity to $\sin ^{2} \theta_{W}$.

Figure 2 depicts the DUNE sensitivity to the vector and axial couplings, $g_{V}$ and $g_{A}$, in the on-axis LArTPC (dashed green) or the DUNE-PRISM configuration (dark blue). For comparison, we include existing measurements from CHARM-II [37] (gray), LSND [38] (dotted light-brown), and TEXONO [39] (dot-dashed light-violet). Both the DUNE on-axis and CHARM-II measurements suffer from a fourfold degeneracy; this is a consequence of the fact that the neutrino flux in both these experiments is dominated by $\nu_{\mu}$. There is an exact degeneracy in the differential cross section for $\nu_{\mu}-e$ scattering under the transformations

$\left(g_{V}, g_{A}\right) \rightarrow\left(g_{A}, g_{V}\right) \quad$ and $\quad\left(g_{V}, g_{A}\right) \rightarrow\left(-g_{V},-g_{A}\right)$,

see Eq. (2) and Table I, and hence an experiment with a pure $\nu_{\mu}$ beam is intrinsically limited. The TEXONO experiment measured electron recoils from electron antineutrinos produced in a nuclear reactor. The scattering cross section, in this case, is proportional to $3 g_{1}^{2}+g_{2}^{2}$, which defines an oblique ellipse in the $\left(g_{V}, g_{A}\right)$ plane centered at $(-0.5,-0.5)$. The TEXONO result in Fig. 2 reflects this fact, up to effects related to information on the recoil energy spectrum. The LSND measurement can also be understood by noticing that the flux of neutrinos consists of $\nu_{\mu}, \bar{\nu}_{\mu}$, and $\nu_{e}$ with well-characterized energy spectra from (mostly) pion decay at rest, followed by muon decay at rest. Current data are not able to rule out very small $g_{A}$ and $g_{V} \sim-0.5$ (region on the left-hand part of Fig. 2). 


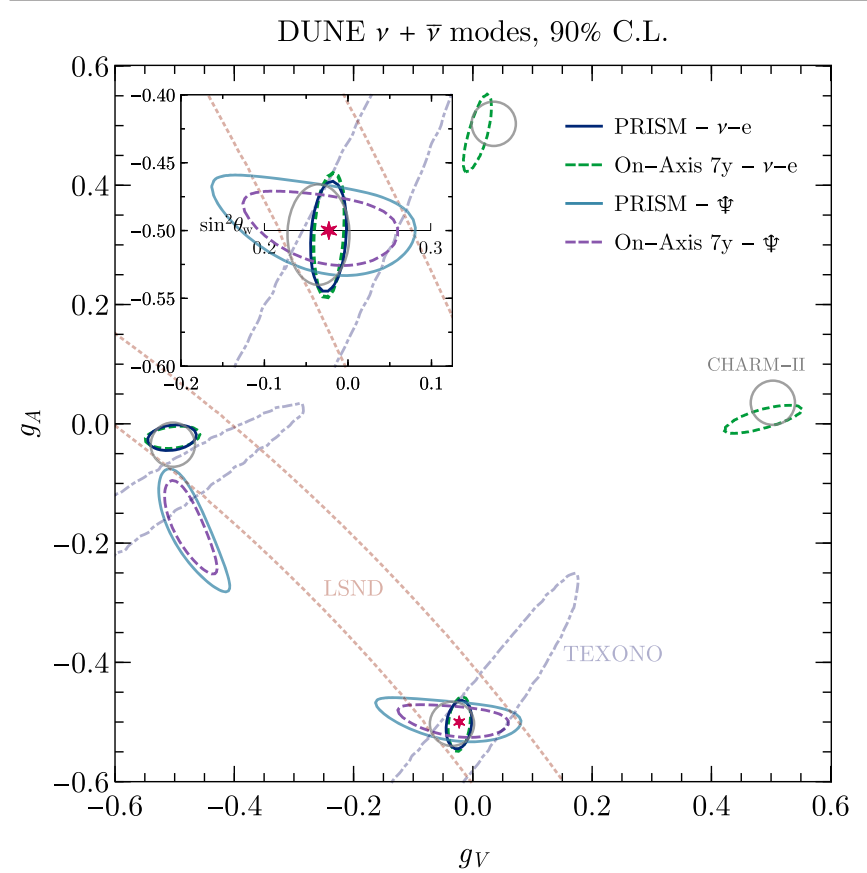

FIG. 2. Allowed regions in the plane $g_{V} \times g_{A}$ from CHARM-II [37] (gray, at 90\% C.L.), LSND [38] (dotted light-brown, at $1 \sigma$ C.L.), and TEXONO [39] (dot-dashed light-violet, at $1 \sigma$ C.L.), and the estimated $90 \%$ C.L. sensitivity from on-axis (7 years) DUNE electron scattering (dashed green) and tridents $(\Psi)$ (dashed purple), as well as DUNE-PRISM electron scattering (dark-blue) and tridents (light-blue), assuming the SM value $\mathrm{z}$ (red star).

In DUNE-PRISM, the presence of both $\nu_{\mu}$ and $\nu_{e}$, along with their antiparticles, is a powerful tool for lifting degeneracies without resorting to data from other experiments. To illustrate this point, Fig. 3 depicts the $E_{e} \theta_{e}^{2}$ spectra of neutrino-electron scattering events for the first 5 off-axis positions without any angular or energy resolution. For each position, histograms corresponding to three pairs of vector and axial couplings $\left(g_{V}, g_{A}\right)$ are depicted: $(-0.02,-0.5)$, the SM expectation (solid); $(-0.48,-0.04)$, the leftmost degenerate region (dotted); and $(0.47,0.01)$, the rightmost degenerate region (dashed). It is clear that the rightmost degeneracy is lifted due to the higher $\nu_{e}$ composition of the flux, as depicted in Fig. 1. Error bars illustrating the statistical and systematic errors, are included for the SM case. DUNEPRISM neutrino-electron scattering data, alone, cannot fully distinguish the SM from the leftmost degenerate region, as depicted in Fig. 2.

Neutrino-trident scattering, when a neutrino scatters off a nucleus producing a charged lepton pair with the same or different flavors, $\nu A \rightarrow \nu \ell^{+} \ell^{-} A$, is also sensitive to $g_{V}$ and $g_{A}$ [47]. This scattering can be coherent off the electromagnetic field of the nucleus or diffractive off the nucleons themselves. Although the trident cross section is quite involved (see, e.g., Refs. [48-50]), in the limit where the final state leptons are massless, it is proportional to the

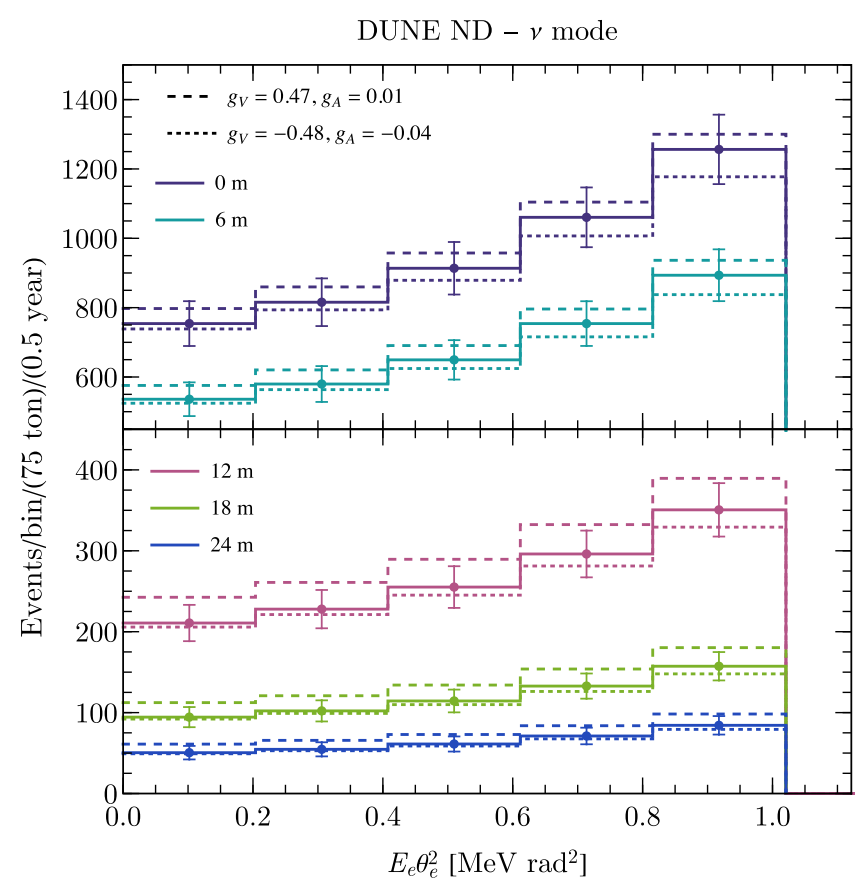

FIG. 3. Neutrino-electron event rates as a function of $E_{e} \theta_{e}^{2}$ for the first 5 off-axis positions. For each position, the three histograms correspond to three pairs of vector and axial couplings $\left(g_{V}, g_{A}\right):(-0.02,-0.5)$ (solid); $(-0.48,-0.04)$ (dotted); and $(0.47,0.01)$ (dashed). Error bars illustrating the statistical and systematic errors are included for the SM case (solid histogram).

electroweak parameters $\left(C_{V}^{2}+C_{A}^{2}\right)$. For a $\nu_{\mu}$ beam, these couplings are $[48,49]$

$$
\begin{gathered}
C_{V}=g_{V} \quad C_{A}=g_{A} \quad\left(e^{+} e^{-} \text {trident }\right), \\
C_{V}=g_{V}+1 \quad C_{A}=g_{A}+1 \quad\left(\mu^{+} \mu^{-} \text {trident }\right) .
\end{gathered}
$$

The processes that lead to $\mu^{\mp} e^{ \pm}$tridents are pure charged current and do not contribute to this discussion. Hence, measurements of $e^{+} e^{-} \nu_{\mu}$-tridents- the statistically dominant mode-constrain $g_{V}^{2}+g_{A}^{2}$, while those of $\mu^{+} \mu^{-} \nu_{\mu^{-}}$ tridents constrain $\left(g_{V}+1\right)^{2}+\left(g_{A}+1\right)^{2}$ in the limit of vanishing muon mass. A similar behavior is expected of $\nu_{e}$ tridents, with $e \leftrightarrow \mu$, and those associated to antineutrinos. It is easy to see that, in the limit where the muon mass vanishes, all cross sections are invariant under $g_{V} \leftrightarrow g_{A}$. A finite muon mass, however, breaks the $g_{V} \leftrightarrow g_{A}$ symmetry.

Because of the very high intensity of the DUNE neutrino beam, this rare process is accessible. Figure 2 also depicts the measurement of $\left(g_{V}, g_{A}\right)$ from both $\mu^{+} \mu^{-}$and $e^{+} e^{-}$ neutrino-trident events in DUNE on axis (dashed purple) and DUNE-PRISM (light-blue), considering the efficiencies from Ref. [49], which range from 48\%-66\% (17\%-39\%) for coherent (diffractive) trident processes. These efficiencies stem from cuts on hadronic activity and kinematical variables in order to make backgrounds negligible. Improvements on 


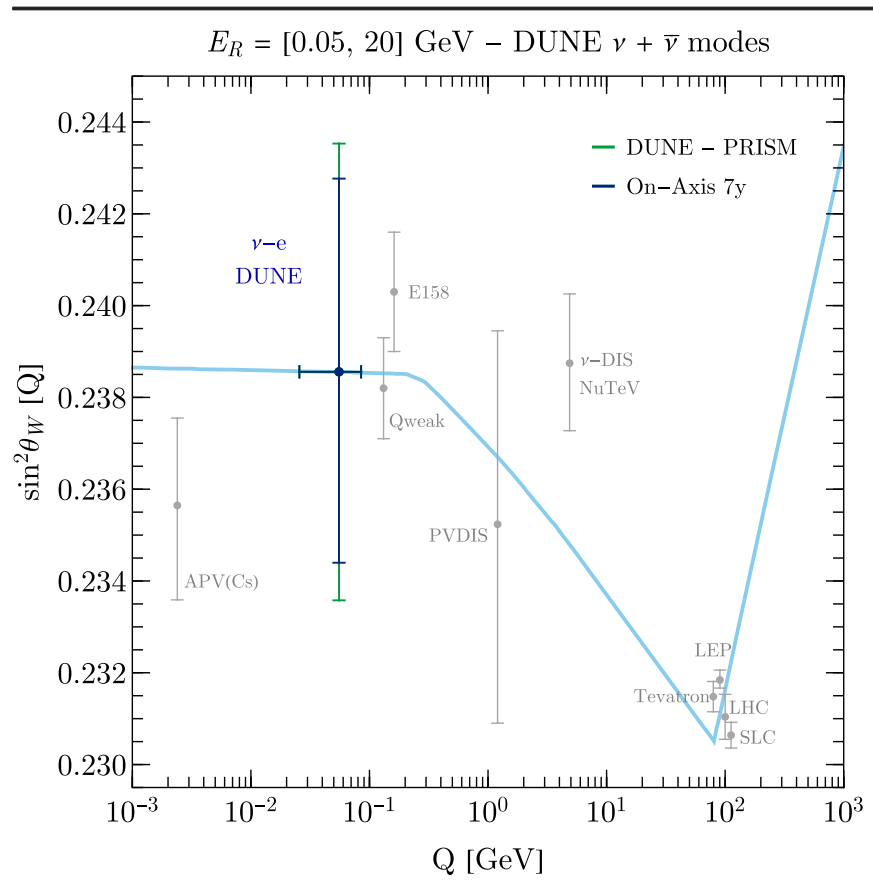

FIG. 4. $\sin ^{2} \theta_{W}$ in the $\overline{\mathrm{MS}}$ scheme (light blue line) as a function of $Q$, obtained from a fit to existing data (gray data points), together with the DUNE on-axis (dark blue data point) and DUNE-PRISM (green data point) sensitivities to this angle. The horizontal error bars indicate the range of $Q$ values accessible to DUNE neutrino-electron scattering. Note that the Tevatron, LHC, and SLC data points were slightly shifted from $Q=M_{Z}$ to improve readability.

the reconstruction of di-electron or di-muon events would benefit the $\left(g_{V}, g_{A}\right)$ couplings determination. The allowed region is not symmetric under $g_{V} \leftrightarrow g_{A}$ since, as highlighted earlier, for DUNE energies, the mass of the muon is not negligible. Indeed, we checked that the subleading $\mu^{+} \mu^{-}$ neutrino-trident event sample plays the decisive role here. Hence, the combination of neutrino-electron scattering and neutrino trident data in the DUNE near-detector complex, assuming these are consistent with the SM, lifts all degeneracies in the $g_{V} \times g_{A}$ plane even if one chooses to exclude information from outside data.

Assuming the SM, our results can be translated into a measurement of $\sin ^{2} \theta_{W}$ at $\left\langle Q^{2}\right\rangle=(55 \mathrm{MeV})^{2}$. Figure 4 depicts the value of $\sin ^{2} \theta_{W}$ in the $\overline{\mathrm{MS}}$ scheme as a function of $Q$, obtained from a fit to existing data, together with our estimate for the expected DUNE and DUNE-PRISM sensitivities. The former is slightly better, but we emphasize that the on-axis measurement of $\sin ^{2} \theta_{W}$ depends more strongly on the neutrino-flux modeling, while the DUNEPRISM sensitivity depends more on the relative on- to offaxis flux uncertainties. The main systematic uncertainty for this analysis comes from hadron production in the beam target, and extra running time would further improve the determination of $\sin ^{2} \theta_{W}$ (see Supplemental Material [45]). Note that current experiments like NA61/SHINE [51] or the future experiment EMPHATIC [52] may achieve a better knowledge of the hadron production mechanism leading to reduced systematic uncertainties and thus improving the determination of the weak mixing angle. Regardless, both measurements are estimated to be competitive with existing results.

In summary, we estimated that the future DUNE experiment will have excellent sensitivity to the vector and axial couplings of the electron to the $Z$ boson, and thus to the weak mixing angle $\sin ^{2} \theta_{W}$, via precision measurements of neutrino-electron scattering. The subdominant $\nu_{e}$ beam component in DUNE-PRISM, as well as neutrino trident events, play an important role in resolving degeneracies currently present in the world data.

We are extremely grateful to Luke Pickering for providing us with the flux covariance matrix, and we thank Laura Fields and Oleksandr Tomalak for useful discussions. The work of A. d. G. is supported in part by the DOE Office of Science Award No. DE-SC0010143. This Letter has been authored by Fermi Research Alliance, LLC under Contract No. DE-AC02- 07CH11359 with the U.S. Department of Energy, Office of Science, Office of High Energy Physics. Z. T. is supported by Fundação de Amparo à Pesquisa do Estado de São Paulo (FAPESP) under Contract No. 2018/ 21745-8.

[1] J. Erler and M. J. Ramsey-Musolf, Phys. Rev. D 72, 073003 (2005).

[2] J. Erler and R. Ferro-Hernández, J. High Energy Phys. 03 (2018) 196.

[3] M. Tanabashi et al. (Particle Data Group), Phys. Rev. D 98, 030001 (2018).

[4] G. P. Zeller, K. S. McFarland, T. Adams, A. Alton, S. Avvakumov et al. (NuTeV Collaboration), Phys. Rev. Lett. 88, 091802 (2002); 90, 239902(E) (2003).

[5] S. Schael et al. (ALEPH, DELPHI, L3, OPAL, SLD Collaborations, LEP Electroweak Working Group, SLD Electroweak Group, and SLD Heavy Flavour Group), Phys. Rep. 427, 257 (2006).

[6] J. Pumplin, D. R. Stump, J. Huston, H. L. Lai, P. M. Nadolsky, and W. K. Tung, J. High Energy Phys. 07 (2002) 012.

[7] S. Kretzer, F. Olness, J. Pumplin, D. Stump, W.-K. Tung, and M. H. Reno, Phys. Rev. Lett. 93, 041802 (2004).

[8] E. Sather, Phys. Lett. B 274, 433 (1992).

[9] E. N. Rodionov, A. W. Thomas, and J. T. Londergan, Mod. Phys. Lett. A 09, 1799 (1994).

[10] A. D. Martin, R. G. Roberts, W. J. Stirling, and R. S. Thorne, Eur. Phys. J. C 35, 325 (2004).

[11] J. T. Londergan and A. W. Thomas, Phys. Rev. D 67, 111901(R) (2003).

[12] W. Bentz, I. C. Cloet, J. T. Londergan, and A. W. Thomas, Phys. Lett. B 693, 462 (2010).

[13] M. Gluck, P. Jimenez-Delgado, and E. Reya, Phys. Rev. Lett. 95, 022002 (2005).

[14] S. Kumano, Phys. Rev. D 66, 111301(R) (2002). 
[15] S. A. Kulagin, Phys. Rev. D 67, 091301(R) (2003).

[16] S. J. Brodsky, I. Schmidt, and J.-J. Yang, Phys. Rev. D 70, 116003 (2004).

[17] M. Hirai, S. Kumano, and T. H. Nagai, Phys. Rev. D 71, 113007 (2005).

[18] G. A. Miller and A. W. Thomas, Int. J. Mod. Phys. A 20, 95 (2005).

[19] I. C. Cloet, W. Bentz, and A. W. Thomas, Phys. Rev. Lett. 102, 252301 (2009).

[20] K. P. O. Diener, S. Dittmaier, and W. Hollik, Phys. Rev. D 69, 073005 (2004).

[21] A. B. Arbuzov, D. Yu. Bardin, and L. V. Kalinovskaya, J. High Energy Phys. 06 (2005) 078.

[22] K. Park, U. Baur, and D. Wackeroth, in Particles and Fields. Proceedings, Meeting of the Division of the American Physical Society, DPF 2009, Detroit, USA (2009), https://arxiv.org/abs/0910.5013.

[23] K. P. O. Diener, S. Dittmaier, and W. Hollik, Phys. Rev. D 72, 093002 (2005).

[24] B. A. Dobrescu and R. K. Ellis, Phys. Rev. D 69, 114014 (2004).

[25] R. Acciarri et al. (DUNE Collaboration), arXiv:1512.06148.

[26] K. Abe et al., arXiv:1109.3262.

[27] L. Alvarez-Ruso et al., Prog. Part. Nucl. Phys. 100, 1 (2018).

[28] J. M. Conrad, J. M. Link, and M. H. Shaevitz, Phys. Rev. D 71, 073013 (2005).

[29] A. de Gouvêa and J. Jenkins, Phys. Rev. D 74, 033004 (2006).

[30] S. K. Agarwalla and P. Huber, J. High Energy Phys. 08 (2011) 059.

[31] J. M. Conrad, M. H. Shaevitz, I. Shimizu, J. Spitz, M. Toups, and L. Winslow, Phys. Rev. D 89, 072010 (2014).

[32] A. Adelmann, J. Alonso, W. A. Barletta, J. M. Conrad, M. H. Shaevitz, J. Spitz, M. Toups, and L. A. Winslow, Adv. High Energy Phys. 2014, 347097 (2014).

[33] K. Abe et al. (T2K Collaboration), Phys. Rev. D 87, 012001 (2013); 87, 019902(A) (2013).

[34] L. Aliaga et al. (MINERvA Collaboration), Phys. Rev. D 94, 092005 (2016); 95, 039903(A) (2017).

[35] C. M. Marshall, K. S. McFarland, and C. Wilkinson, Phys. Rev. D 101, 032002 (2020).
[36] T. Cai, J. Calcutt, K. Mahn, L. Pickering, S. Manly, H. Tanaka, C. Vilela, M. Wilking, and G. Yang, The DUNEPRISM Near Detector (2018), https://indico.fnal.gov/event/ 16205/contribution/2/material/0/0.pdf.

[37] P. Vilain et al. (CHARM-II Collaboration), Phys. Lett. B 335, 246 (1994).

[38] L. B. Auerbach, R. L. Burman, D. O. Caldwell, E. D. Church, J. B. Donahue et al. (LSND Collaboration), Phys. Rev. D 63, 112001 (2001).

[39] M. Deniz et al. (TEXONO Collaboration), Phys. Rev. D 81, 072001 (2010).

[40] M. Carena, A. de Gouvêa, A. Freitas, and M. Schmitt, Phys. Rev. D 68, 113007 (2003).

[41] O. Tomalak and R. J. Hill, Phys. Rev. D 101, 033006 (2020).

[42] R. J. Hill and O. Tomalak, Phys. Lett. B 805, 135466 (2020).

[43] We have checked numerically that flavor-dependent electroweak corrections do not change our results at the $1 \sigma$ level.

[44] L. Pickering (private communication).

[45] See Supplemental Material at http://link.aps.org/ supplemental/10.1103/PhysRevLett.125.051803 for further discussions on the technical details of the analysis: the event rates per channel for both neutrino-electron and trident scatterings, the neutrino fluxes considered, as well as the impact of different angular resolutions and running plans on the measurement of the mixing angle.

[46] T. Golan, C. Juszczak, and J. T. Sobczyk, Phys. Rev. C 86, 015505 (2012).

[47] Here we assume that the electron and muon couplings to the $Z$ boson are identical.

[48] G. Magill and R. Plestid, Phys. Rev. D 95, 073004 (2017).

[49] P. Ballett, M. Hostert, S. Pascoli, Y. F. Perez-Gonzalez, Z. Tabrizi, and R. Zukanovich Funchal, J. High Energy Phys. 01 (2019) 119.

[50] W. Altmannshofer, S. Gori, J. Martín-Albo, A. Sousa, and M. Wallbank, Phys. Rev. D 100, 115029 (2019).

[51] N. Abgrall et al. (NA61/SHINE Collaboration), Phys. Rev. C 84, 034604 (2011).

[52] T. Akaishi et al. (EMPHATIC Collaboration), arXiv:1912 .08841 . 